measurements as a function of temperature or otherwise to adjust the viscosity. The presence of free conjugate in the solution does not interfere with the determina. tions, and it should therefore be possible to use adsorbed instead of chemically coupled dyes. These advantages should widen the scope of an increasingly popular technique for the study of processes involving binding or shape changes in proteins.

At the conference, extensive data were given by Brahms on the stacking of dinucleotides in aqueous salt solutions. Contrary to the conclusions of earlier workers, all dinucleotide phosphates at neutral $p H$ show stacking and differ only in the magnitude of the equilibrium constant. Enthalpies of stacking are in all cases similar. The data have been applicd to the analysis of the conformation of a specific transfer RNA.

\section{Cancer by Viruses}

\section{from a Correspondent in Cell Biology}

Some elegantly simple experiments of Defendi, Ephrussi et al. (Proc. U.S. Nat. Acad. Sci., 5\%, 299; 1967) have provided strong support for the general hypothesis that cancer induced by virus results from the addition of genetic information.

Uncontrolled growth is the characteristic property that distinguishes all cancer cells from normal cells. Cancer colls are unable to regulate cell division and, since this characteristic is inherited, it is highly likely that it results from a genetic change. Before the discovery that DNA and RNA viruses can both transform normal cells into cancer cells, the hypothesis most frequently considered was that cancer is caused by the accumulation of somatic mutations leading to the loss of the function of some essential regulatory gene. With the discovery of eancer inducing or oncogenic viruses, an alternative hypothesis could be considered - that cancer results from the acquisition of genetic information. Of course, it is equally possible that oncogenic viruses either cause a deletion of part of the cell genome or induce recessive mutations. The crucial question then is whether the genome of an oncogenic virus persists in transformed cells, pcrhaps incorporated into the chromosome as a prophage is in a bacterium.

Defendi, Ephrussi and their colleagues have now produced hybrid cells by growing, in the same culture, normal mouse cells and mouse cells which have been transformed to cancer cells by polyoma virus. The question is whether the hybrids have the properties of the normal cells or of the transformed cells. The answer is that they clearly have the three characteristics of the polyoma transformed parental cellsthey produce polyoma induced transplantation and complement fixing antigens (which are not made by normal mouse cells), they are neoplastic and, when injected into young mice, they induce tumours. This result is incompatible with the hypothesis that viral induced cancer results from recessive mutations or the deletion of genetic material, becausc the hybrid cells contain the normal genome donated by the parental normal cells as well as the genome of the parental transformed cells. Moreover, the same experiments show that the hybrids are more tumorigenic than original parental polyoma transformed cells. Whatever the reason, and Defendi, Ephrussi et al. discuss several possibilities, it does seem that in vivo the fusion of tumour cells with adjacent normal cells may be the cause of tumour progression.

This result is in agreement with those of Benjamin (1966), who showed a small fraction of the RNA in cells transformed by polyoma and which are free of virus particles and can be hybridized with polyoma DNA. It is difficult to interpret this observation in any other way than that the polyoma genome persists in transformed cells.

\section{Collagen Meeting}

Ат a one-day meeting of scientists working on collagen at the British Leather Manufacturers' Research Association last week, it was decided to set up a Collagen Group to stimulate discussion and to hold meetings at appropriate centres. Mectings of the group are likely to be timed to coincide with visits of scientists from abroad, who could then describe their work. For the time being, the group decided to remain independent of the Biochemical Society.

Dr. K. Piez of the U.S. National Institutes of Health described recent amino-acid sequence analysis on the collagen molecule; it was, he said, a rod-like molecule containing three chains, two $\alpha_{1}$ chains and one $\alpha_{2}$ chain. Breaking up the molecule into smaller pieces by cleavage, for example, with cyanogen bromide had made it possible to determine the order of amino-acid residues in the $\alpha_{1}$ chain in rat skin collagen. Eight unique peptides in the $\alpha_{1}$ chain had been demonstrated by chromatography, and smaller satellite peaks in the chromatograms did not indicate two kinds of $\alpha_{1}$ chain. They were either artefacts, or minor variants, and both $\alpha_{1}$ chains were identical, or nearly so. All but the first peptide had glycine as every third residuc.

Dr. Piez also described a difference between rat skin and rat tail tendon collagen; residues 16 to 51 in the $\alpha_{1}$ chain showed different quantities of proline and hydroxyproline; either some positions interchange, or hydroxylation is incomplete in this part of the molecule. The difference in chemistry, Dr. Piez suggested, might be related to the difference in function between skin and tail tendon.

At the same meeting, Dr. J. H. Bowes of the research association described the interaction of aldehydes with collagen. Little was known about the insoluble fraction in collagen, but she described the process of tanning leather as tying up the cross-links broken by the previous treatment of the hide. Glutaraldehyde was the most effective cross-linking agent, and acrolein was also good. At physiological $p \mathrm{H}$, aldehydes would form cross-links with amino groups, but the precise chemistry of the links was still obscure.

\section{School Mathematics}

Professor K. W. Keohane writes:

Mathematics holds a special place in the school curriculum. Few would dispute that in Britain young pcople are not given encouragement to continue the subject as far as many of them could. But once mathematics has been dropped, people are lost forever to the physical sciences and technology-and increasingly to the biological sciences as well. For those who do continue, however, the mathematics they are taught is all too often inappropriate, mechanical and dull. This was the background for the splendid 